\title{
A novel beam focus control at the entrance to the ANU 14UD accelerator
}

\author{
M. De Cesare ${ }^{1, a}$, D. C. Weisser ${ }^{1}$, L. K. Fifield ${ }^{1}$, T. B. Tunningley ${ }^{1}$, and N.R. Lobanov ${ }^{1}$ \\ ${ }^{1}$ Department of Nuclear Physics, Research School of Physics and Engineering, Australian National University, Canberra, \\ ACT 0200, Australia
}

\begin{abstract}
Tandem electrostatic accelerators often require the flexibility to operate at variety of terminal voltages to cater for various user needs. However beam transmission will only be optimal for a limited range of terminal voltages. This paper describes a focussing system that greatly expands the range of terminal voltages for optimal transmission. This is achieved by controlling the gradient of the entrance of the low-energy tube providing an additional controllable focusing element. Up to $150 \mathrm{kV}$ is applied to the fifth electrode of the first unit of the accelerator tube giving control of the tube entrance lens strength. Beam tests to confirm the efficacy of the lens have been performed. These tests demonstrate that the entrance lens control eliminates the need to short out sections of the tube for low terminal voltage operation.
\end{abstract}

\section{Introduction}

The beam optical effects at the entrance to the low-energy tube of an accelerator are crucial to transport beam though it. This focussing effect at the tube entrance is due to the transition in voltage gradient [1]. The lens strength is proportional to the ratio of the voltage gradient on the accelerator tube to the injection beam energy [2]. Accelerators use additional lenses preceding the tube entrance lens, to focus the beam through the stripper canal in the high voltage terminal.

The Australian National University (ANU) 14UD accelerator requires terminal variability from $15.5 \mathrm{MV}$ down to $2 \mathrm{MV}$ to cater to the wide range of nuclear physics and accelerator mass spectrometry (AMS) applications. Optimum beam transmission can only be achieved within $\sim 20 \%$ of the maximum terminal voltage. At lower voltages, shorting rods have been used to achieve better transmission as is the case at many other tandem accelerator facilities. A series of steel and nylon rods are used to maintain the tube entrance gradient near that typical at full terminal voltage, i.e. $\sim 23 \mathrm{kV} / \mathrm{cm}$.

For the low-energy tube, the steel rods short together accelerator units adjacent to the terminal leaving the ones at the entrance, spanned by the nylon rods, close to the nominal gradient value.

The insertion and removal of shorting rods risks the loss of the sulphur hexafluoride $\left(\mathrm{SF}_{6}\right)$ insulating gas and so is restricted to a few experienced technical staff members. Consequently, shorting rod configuration changes are only allowed during normal working hours which curtails the flexibility experimenters enjoy. In reference [3] a solution was presented for the control of the voltage gradient at the low-energy tube entrance using an external power supply. A $+150 \mathrm{kV}$ Glassman, PS/LT150P010-22, power supply provides an adjustable voltage to the fifth tube electrode via a tank wall feed-through. This power supply not only maintains the focusing effect of the tube but also provides the opportunity to tune the beam by adjusting the entrance lens. The feed-through includes a $10 \Omega$ series protection resistor and a pair of spark-gaps designed to fire at $\sim 180 \mathrm{kV}$ in 7 atmosphere $\mathrm{SF}_{6}$ pressure. The high voltage feed-through has been tested successfully at $150 \mathrm{kV}$. In the present paper we present beam optics calculations for terminal voltages of 14, 10, 6 and $4 \mathrm{MV}$ with and without the shorting rods and with lens on at $150 \mathrm{kV}$ and off. Further simulation results are obtained for $4 \mathrm{MV}$ with a range of lens voltages. Beam transmission tests at various terminal voltages are also presented. Different lens settings are needed for different terminal voltages.

\section{Upgrade of the ANU accelerator}

The 14UD ANU tandem accelerator has been described elsewhere [4]. As high energy is required to exploit certain techniques of isobar separation effectively in AMS research, this makes the ANU tandem well suited for research based on heavier isotopes e.g., ${ }^{36} \mathrm{Cl}$ and ${ }^{60} \mathrm{Fe}$. When lower energy is necessary as for ${ }^{236} \mathrm{U}$ and ${ }^{\mathrm{x}} \mathrm{Pu}$ isotopes, sections of the accelerator tube can be shorted out, in order to optimise the ion optics for maximum transmission.

\footnotetext{
a Corresponding author: mario.decesare@anu.edu.au
} 


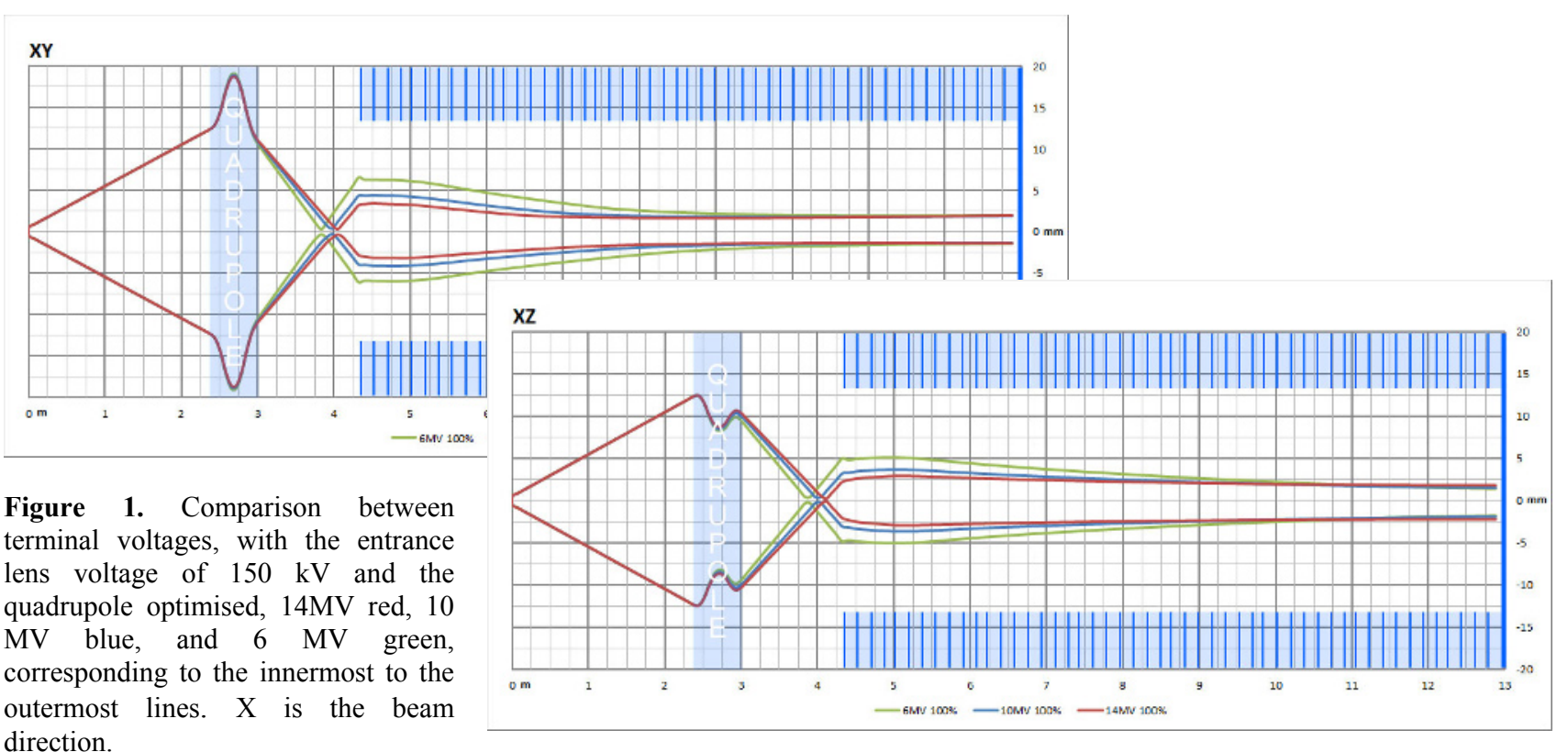

The AMS program uses a caesium sputter ion source -32sample MC-SNICS (Multi Cathode Source for Negative Ions by Caesium Sputtering) manufactured by National Electrostatics Corporation. Negative ions are injected with energies between $105-155 \mathrm{keV}$ and then mass selected by the $90^{\circ}$ double focusing magnet. An electrostatic quadrupole triplet incorporating steerers places the ions on an optimum trajectory for injection into the accelerator.

In June-July 2012, the high voltage feed-through was installed to provide adjustable voltage up to $+150 \mathrm{kV}$ to the fifth electrode of the first unit of the accelerator tube. This provides a voltage gradient of up to $30 \mathrm{kV} /$ gap (a gap is $1.32 \mathrm{~cm}$ ) which is the nominal voltage gradient at 15.5 MV. For terminal voltages above $12 \mathrm{MV}$, this additional power supply does not play any role since the entrance gradient associated with these terminal voltages is close to optimal. However at $10 \mathrm{MV}$ the voltage gradient is only $19.3 \mathrm{kV} / \mathrm{gap}$, and decreases with the terminal voltage: i.e. $11.6 \mathrm{kV} /$ gap for $6 \mathrm{MV}$ and 7.7 $\mathrm{kV} /$ gap for $4 \mathrm{MV}$. For such terminal voltages the shorting rods have been used in the past to maintain the desirable transmission; e.g. 4 steel rods and 10 nylon are used in the case of $10 \mathrm{MV} ; 8$ steel rods and 6 nylon in the 6 and 4 MV cases. The voltage gradient with shorting rods at 10 and $6 \mathrm{MV}$ is 27.0 and is $18.0 \mathrm{kV} / \mathrm{gap}$ for $4 \mathrm{MV}$.

\section{Simulations and experimental results}

\subsection{Low-energy tube lens}

Simulations show that the beam transmission, when fixing the entrance gradient at $30 \mathrm{kV} / \mathrm{gap}$, should be at least as good as achieved with shorting rods. The simulations take the beam from the image slits of the injection magnet to the terminal using SIMION [5]. To obtain clear comparisons between different gradient configurations, ions were launched at $0.05 \mathrm{~mm}$ steps between -0.5 and $+0.5 \mathrm{~mm}$ from the central ray along lines on both the $\mathrm{Y}$ and $\mathrm{Z}$ axes. At each position, the angle was varied between -2.5 and $+2.5 \mathrm{mrad}$ in steps of $0.25 \mathrm{mrad}$, (Note that in the previous paper [3] a different approach was employed. The beam at the slits comprised 200 particles dispersed in a Gaussian distribution with a sigma of 0.2 $\mathrm{mm}$. A uniform angular distribution between $\pm 2.5 \mathrm{mrad}$ was assumed at each particle launch point. Due to the random nature of the spatial distribution, however, no two runs gave identical results which made it difficult to compare different configurations). The components modelled were an electrostatic quadrupole triplet lens, the accelerator tubes in the first two of the 14 units of the 14UD using complete details of the electrode geometry, followed by twelve units of simplified, planar electrodes, for the remaining tube to the terminal. The simplified electrode structure was used to reduce computer memory requirements and to reduce computing time. The results of the simulations are in Table 1 . The gradient at $14 \mathrm{MV}$ intrinsically is $27 \mathrm{kV} / \mathrm{gap}$ but only a slight difference in focussing is observed when the electrostatic triplet is reoptimised for the entrance lens setting of $30 \mathrm{kV} / \mathrm{gap}$. Figure 1 shows that the use of the tube entrance lens maintains focusing comparable to that at $14 \mathrm{MV}$ for terminal voltages of 10 and $6 \mathrm{MV}$. Moreover Table 1 shows that the use of the shorting rods rather than the entrance lens does not improve the focusing and results in an asymmetric beamspot. Note that, for the $6 \mathrm{MV}$ case, the optimal focusing is obtained when an entrance lens voltage of $80 \%$ of the $150 \mathrm{keV}$ injection energy is applied instead of the $100 \%$ setting. There is no additional gain from running at lower lens voltages. This conclusion has been confirmed in the beam tests, see paragraph 3.2. Figure 1 also shows that the beam envelope increases in the first tube sections with decreasing terminal voltage as a consequence of the first waist moving closer to the quadrupole triplet lens. The beam envelope also increases for a fixed terminal voltage, when lower strengths of the lens are used as seen in Figure 2, where lens voltages $120 \%, 100 \%$ and $80 \%$ of the injection energy for $4 \mathrm{MV}$ terminal voltages are compared. Table 1 shows, for the 4 MV case and but at $70 \%$ lens voltage, the beam is 


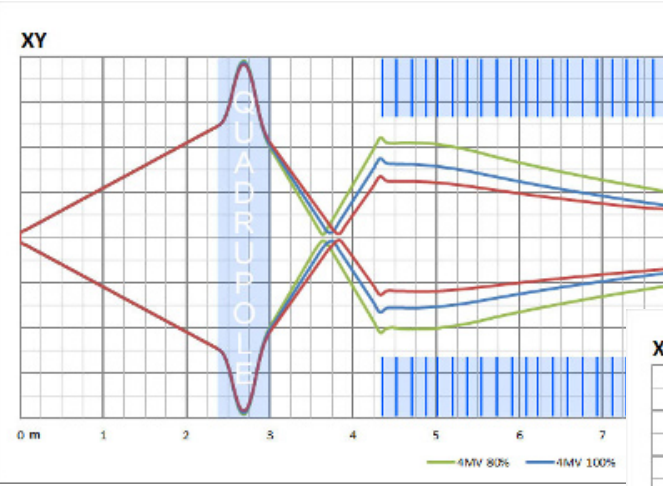

Figure 2. Comparison at $4 \mathrm{MV}$ terminal voltage, and $150 \mathrm{keV}$ injection energy for different lens voltages: $120 \%$ red, $100 \%$ blue and $80 \%$ green as percentage of the injection energy, corresponding to the innermost to the outermost lines at the tube entrance. $\mathrm{X}$ is the beam direction.

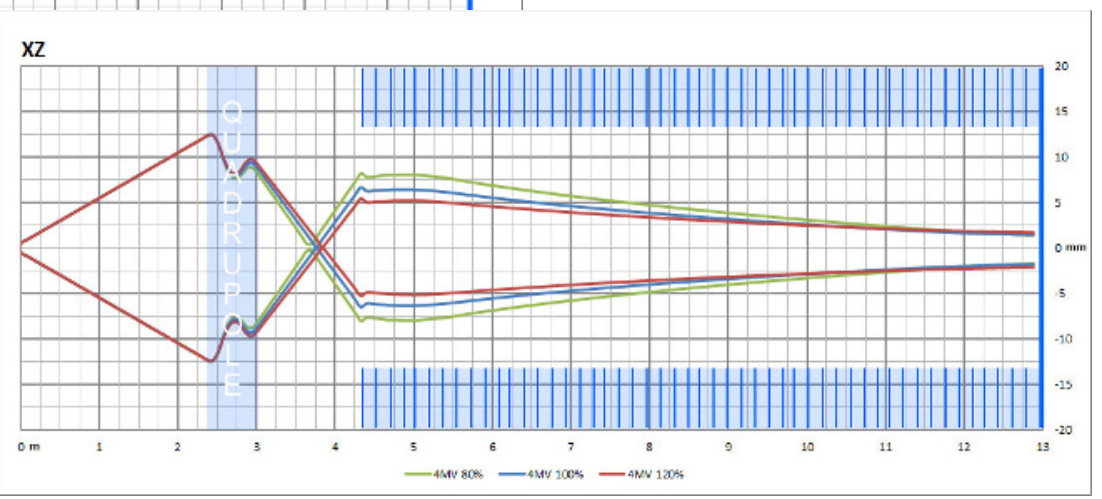

\begin{tabular}{|c|c|c|c|c|}
\hline $\begin{array}{l}\text { TV } \\
\text { (MV) }\end{array}$ & $\begin{array}{c}\mathbf{E}_{\mathrm{inj}} \\
(\mathbf{k e V})\end{array}$ & $\begin{array}{c}E L \\
\left(\% \Delta V_{\text {inj }}\right) \\
\text { or } \\
\text { SR }\end{array}$ & $\begin{array}{c}\text { EQ Out/In } \\
(\mathrm{kV})\end{array}$ & $\begin{array}{c}\text { Waist } \\
\mathrm{XY} / \mathrm{XZ} \\
(\mathrm{mm})\end{array}$ \\
\hline \multirow{2}{*}{14} & \multirow{2}{*}{150} & - & $4.10 / 4.42$ & $3.6 / 3.6$ \\
\hline & & $100 \%$ & $4.07 / 4.40$ & $3.4 / 4.0$ \\
\hline \multirow{3}{*}{10} & \multirow{3}{*}{150} & SR & $4.18 / 4.49$ & $3.2 / 1.8$ \\
\hline & & $100 \%$ & $4.18 / 4.50$ & $3.3 / 3.5$ \\
\hline & & $80 \%$ & $4.20 / 4.52$ & $3.5 / 3.1$ \\
\hline \multirow{3}{*}{6} & \multirow{3}{*}{150} & SR & $4.24 / 4.54$ & $3.6 / 1.7$ \\
\hline & & $100 \%$ & $4.38 / 4.68$ & $3.4 / 3.2$ \\
\hline & & $80 \%$ & $4.47 / 4.76$ & $3.2 / 2.8$ \\
\hline \multirow{5}{*}{4} & \multirow{5}{*}{150} & $120 \%$ & $4.42 / 4.71$ & $4.1 / 4.8$ \\
\hline & & $100 \%$ & $4.57 / 4.85$ & $3.7 / 3.3$ \\
\hline & & $90 \%$ & $4.65 / 4.92$ & $3.6 / 3.2$ \\
\hline & & $80 \%$ & $4.75 / 5.01$ & $3.4 / 3.1$ \\
\hline & & $70 \%$ & $4.87 / 5.12$ & $3.0 / 3.0$ \\
\hline \multirow{5}{*}{4} & \multirow{5}{*}{100} & $120 \%$ & $2.90 / 3.09$ & $2.5 / 1.6$ \\
\hline & & $100 \%$ & $2.92 / 3.12$ & $3.4 / 3.2$ \\
\hline & & $90 \%$ & $2.94 / 3.14$ & $3.3 / 3.1$ \\
\hline & & $80 \%$ & $2.96 / 3.16$ & $3.3 / 4.0$ \\
\hline & & $70 \%$ & $3.01 / 3.20$ & $3.1 / 2.7$ \\
\hline
\end{tabular}

Table 1. Waist comparison in the $X Y$ and $X Z$ planes at the stripper canal for a range of terminal voltages (TV) and injection energies $\left(\mathrm{E}_{\text {inj }}\right)$, using either the new entrance lens (EL, settings in $\% \Delta \mathrm{V}_{\text {inj }}$ of the injection potential) or shorting rods (SR). The settings for the electrostatic quadrupole triplet lens (EQ) have been optimised in each case. uncomfortably close to the tube electrodes.

Beam transmission for $4 \mathrm{MV}$ terminal voltage is better for injection energy of $100 \mathrm{keV}$ and a lens voltage of about $120 \%$ of the injection energy. In Figure 3, at 100 $\mathrm{keV}$ beam energy, lens voltages $120 \%, 100 \%$ and $80 \%$ of the injection energy, for $4 \mathrm{MV}$ terminal voltage are compared. Note that the simulations display a range of beam sizes in the XZ plane when combined with compensating settings for the electrostatic quadrupole lens.

\subsection{Beam Test}

Beam transmission tests have confirmed the efficacy of the lens in providing somewhat superior transmission to that achieved with shorting rods. It was convenient to use ${ }^{28} \mathrm{Si}^{8+}$ beam as a surrogate for the ${ }^{36} \mathrm{Cl}$ beam. The terminal voltage was $12.7 \mathrm{MV}$, the injection energy was $155 \mathrm{keV}$, and a lens strength of $82 \%(127 \mathrm{kV})$ was used. Gas stripping was employed.

$\mathrm{A}^{28} \mathrm{Si}^{-}$current of $160 \mathrm{nA}$ was injected. The beam at the exit of the accelerator was $540 \mathrm{nA}$ implying a transmission of $54 \%$ assuming a calculated average charge of $6.2 .11 \%$ of the injected beam, $150 \mathrm{nA}$, was measured in the $8+$ charge state. The transmitted beam intensity was not increased by increasing the lens strength to $100 \%$ of the injection energy but it did decrease for lower entrance lens voltages. The lens displays a broad range of acceptable values when combined with compensating settings for the electrostatic quadrupole lens that just precedes the accelerator entrance.

Moreover test transmission for actinides ( $T V \sim 4$ MV) with and without the shorting rods, has demonstrated that transmission is marginally better without rods and with an entrance tube lens at $110 \mathrm{kV}$. The transmission is better at $100 \mathrm{keV}$ injection energy rather than $150 \mathrm{keV}$ with either shorting rods or with the tube entrance lens.

Typical optimal lens voltages are about $80-100 \%$ of the injection energy for high/medium terminal voltages and about $110 \%$ of the injection energy for terminal voltage around $4 \mathrm{MV}$ if lower injection energy (100 keV) is chosen. 


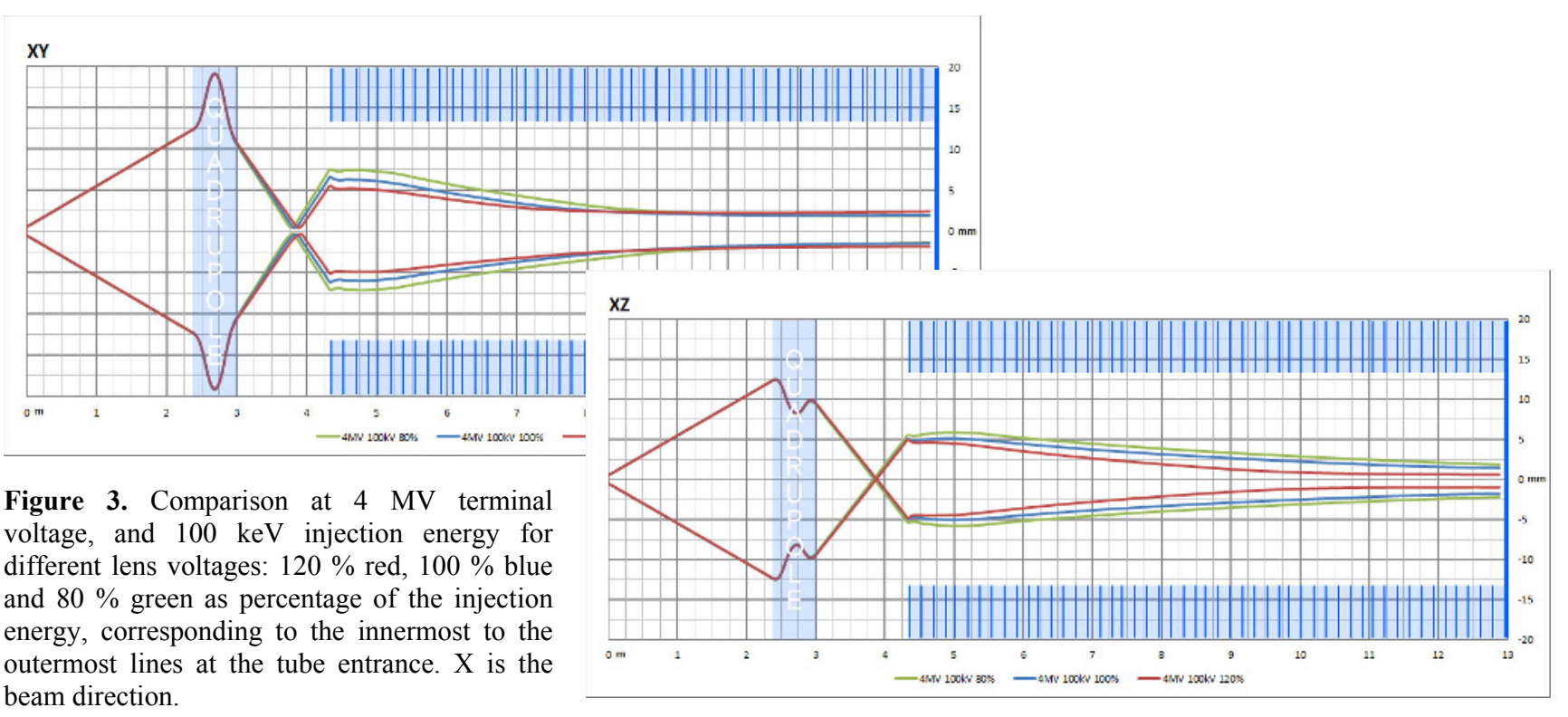

\section{Summary}

A system that controls the entrance tube focusing of the low-energy tube of the 14UD accelerator has been presented. Its use eliminates the need for shorting rods to accommodate low terminal voltage operation which eliminates the risk of the loss of $\mathrm{SF}_{6}$ and allows out-ofhours large changes to the terminal voltage. This entrance lens control not only maintains the focusing effect of the lens, but also provides the opportunity to tune the beam by adjusting its strength. Simulations, confirmed by beam transmission measurements, have shown the effectiveness of the lens compared to the use of shorting rods for a wide range of terminal voltages. A lens setting of $100 \%$ of the injection energy should be used for high terminal voltage operation and injection energy of $150 \mathrm{keV}$. For medium terminal voltage a slight improvement has been obtained by running at $80 \%$ of injection energy $(150 \mathrm{keV})$. For low terminal voltage a better transmission is achieved using $100 \mathrm{keV}$ injection energy and with the lens voltage at 110 $\%$ of the injection energy.

\section{References}

1. J. D. Larson, Nucl. Inst. and Meth. 122, 53 (1974)

2. C. J. Davisson and C. J. Calbick, Phys. Rev. 42, 580 (1932)

3. D. C. Weisser, L. K. Fifield, T. F. G. Kitchen, T. B. Tunningley, N. R. Lobanov, A. G. Muirhead, AIP Conf. Proc. 1515, 506 (2013)

4. L.K. Fifield, R.G. Cresswell, M.L. di Tada, T.R. Ophel, J.P. Day, A.P. Clacher, S.J. King, N. D Priest, Nucl. Instr. Meth. Phys. Res. B 117, 295 (1996)

5. D. A. Dahi, Int. J. Mass Spectrom. 200, 3 (2000) 